KYUNGPOOK Math. J. 55(2015), 597-614

http://dx.doi.org/10.5666/KMJ.2015.55.3.597

pISSN 1225-6951 eISSN 0454-8124

(C) Kyungpook Mathematical Journal

\title{
Some Properties of the Generalized Apostol Type Hermite- Based Polynomials
}

\author{
Waseem Ahmad Khan \\ Department of Mathematics, Integral University, Lucknow-226026, India \\ e-mail : waseem08_khan@rediffmail.com
}

Abstract. In this paper, we study some properties of the generalized Apostol type Hermite-based polynomials. which extend some known results. We also deduce some properties of the generalized Apostol-Bernoulli polynomials, the generalized Apostol-Euler polynomials and the generalized Apostol-Genocchi polynomials of high order. Numerous properties of these polynomials and some relationships between $F_{n}^{(\alpha)}(x ; \lambda ; \mu, \nu, c)$ and ${ }_{H} F_{n}^{(\alpha)}(x, y ; \lambda ; \mu, \nu, c)$ are established. Some implicit summation formulae and general symmetry identities are derived by using different analytical means and applying generating functions.

\section{Introduction}

The generalized Bernoulli polynomials $B_{n}^{(\alpha)}(x)$ of order $\alpha \epsilon C$, the generalized Euler polynomials $E_{n}^{(\alpha)}(x)$ of order $\alpha \epsilon C$ and the generalized Genocchi polynomials $G_{n}^{(\alpha)}(x)$ of order $\alpha \epsilon C$, each of degree $\mathrm{n}$ as well as in $\alpha$, are defined respectively by the following generating function (see,[1, vol.3.p.253 et seq.], [23, Section 2.8] and $[16])$ :

$$
\begin{aligned}
& \left(\frac{t}{e^{t}-1}\right)^{\alpha} e^{x t}=\sum_{n=0}^{\infty} B_{n}^{(\alpha)}(x) \frac{t^{n}}{n !},\left(|t|<2 \pi, 1^{\alpha}=1\right) \\
& \left(\frac{2}{e^{t}+1}\right)^{\alpha} e^{x t}=\sum_{n=0}^{\infty} E_{n}^{(\alpha)}(x) \frac{t^{n}}{n !},\left(|t|<\pi, 1^{\alpha}=1\right)
\end{aligned}
$$

Received November 10, 2014; accepted May 13, 2015.

2010 Mathematics Subject Classification: 11B68, Secondary 33C65.

Key words and phrases: Hermite polynomials, generalized Apostol type Hermite-based polynomials, summation formulae, symmetric identities. 
and

$$
\left(\frac{2 t}{e^{t}+1}\right)^{\alpha} e^{x t}=\sum_{n=0}^{\infty} G_{n}^{(\alpha)}(x) \frac{t^{n}}{n !},\left(|t|<\pi, 1^{\alpha}=1\right)
$$

The literature contains a large number of interesting properties and relationships involving these polynomials $[1,4,5,11,12,24]$. Q.M. Luo and Srivastava([18,20]) introduced the generalized Apostol-Bernoulli polynomials $B_{n}^{(\alpha)}(x)$ of order $\alpha$, Q.M. Luo [15] investigated the generalized Apostol-Euler polynomials $E_{n}^{(\alpha)}(x)$ of order $\alpha$ and the generalized Apostol-Genocchi polynomials $G_{n}^{(\alpha)}(x)$ of order $\alpha$ (see also $[16,17,19])$.

The generalized Apostol-Bernoulli polynomials $B_{n}^{(\alpha)}(x ; \lambda ;)$ of order $\alpha \epsilon C$, the generalized Apostol-Euler polynomials $E_{n}^{(\alpha)}(x ; \lambda)$ of order $\alpha \epsilon C$ and the generalized Apostol-Genocchi polynomials $G_{n}^{(\alpha)}(x ; \lambda)$ of order $\alpha \epsilon C$, are defined respectively by the following generating function

$$
\begin{aligned}
& \left(\frac{t}{\lambda e^{t}-1}\right)^{\alpha} e^{x t}=\sum_{n=0}^{\infty} B_{n}^{(\alpha)}(x ; \lambda) \frac{t^{n}}{n !},\left(|t+\ln \lambda|<2 \pi, 1^{\alpha}=1\right) \\
& \left(\frac{2}{\lambda e^{t}+1}\right)^{\alpha} e^{x t}=\sum_{n=0}^{\infty} E_{n}^{(\alpha)}(x ; \lambda) \frac{t^{n}}{n !},\left(|t+\ln \lambda|<\pi, 1^{\alpha}=1\right)
\end{aligned}
$$

and

$$
\left(\frac{2 t}{\lambda e^{t}+1}\right)^{\alpha} e^{x t}=\sum_{n=0}^{\infty} G_{n}^{(\alpha)}(x ; \lambda) \frac{t^{n}}{n !},\left(|t+\ln \lambda|<\pi, 1^{\alpha}=1\right)
$$

It is easy to see that

$$
B_{n}^{(\alpha)}(x)=B_{n}^{(\alpha)}(x ; 1), E_{n}^{(\alpha)}(x)=E_{n}^{(\alpha)}(x ; 1) \operatorname{and} G_{n}^{(\alpha)}(x)=G_{n}^{(\alpha)}(x ; 1)
$$

In $[8,9]$ Srivastava et al have investigated some new classes of Apostol-Bernoulli, Apostol-Euler and Apostol-Genocchi polynomials with parameters a,b and c defined by the following generating functions.

Definition 1.1. Let $a, b, c \in R^{+}, a \neq b$ and $n \in N_{0}$. The generalized Apostol-Bernoulli polynomials $B_{n}^{(\alpha)}(x ; \lambda ; a, b, c)$ of order $\alpha$, the generalized Apostol-Euler polynomials $E_{n}^{(\alpha)}(x ; \lambda ; a, b, c)$ of order $\alpha$ and the generalized generalized Apostol-Genocchi polynomials $G_{n}^{(\alpha)}(x ; \lambda ; a, b, c)$ of order $\alpha$ are defined respectively by the following generating functions

$$
\left(\frac{t}{\lambda b^{t}-a^{t}}\right)^{\alpha} c^{x t}=\sum_{n=0}^{\infty} B_{n}^{(\alpha)}(x ; \lambda ; a, b, c) \frac{t^{n}}{n !},\left(\left|t \ln \left(\frac{a}{b}\right)+\ln \lambda\right|<2 \pi, 1^{\alpha}=1\right)
$$




$$
\left(\frac{2}{\lambda b^{t}+a^{t}}\right)^{\alpha} c^{x t}=\sum_{n=0}^{\infty} E_{n}^{(\alpha)}(x ; \lambda ; a, b, c) \frac{t^{n}}{n !},\left(\left|t \ln \left(\frac{a}{b}\right)+\ln \lambda\right|<\pi, 1^{\alpha}=1\right)
$$

and

$$
\left(\frac{t}{\lambda b^{t}+a^{t}}\right)^{\alpha} c^{x t}=\sum_{n=0}^{\infty} G_{n}^{(\alpha)}(x ; \lambda ; a, b, c) \frac{t^{n}}{n !},\left(\left|t \ln \left(\frac{a}{b}\right)+\ln \lambda\right|<\pi, 1^{\alpha}=1\right)
$$

If we take $a=1, b=c=e$ in (1.7), (1.8) and (1.9) respectively, we have (1.4), (1.5) and (1.6). Obviously when we set $\lambda=1, \alpha=1, \mathrm{~b}=\mathrm{c}=\mathrm{e}$ in (1.7), (1.8) and (1.9), we have classical Bernoulli polynomials $B_{n}(x)$, classical Euler polynomials $G_{n}(x)$ and classical Genocchi polynomials $G_{n}(x)$.

Recently, Luo and Srivastava [2] introduced a unification (and generalization) of the above-mentioned three families of the generalized Apostol type polynomials.

Definition 1.2. The generalized Apostol type polynomials $F_{n}^{(\alpha)}(x ; \lambda ; \mu, \nu)$ $\left(\alpha \epsilon N_{0}, \mu, \nu \epsilon C\right)$ of order $\alpha$ are defined by means of the following generating function

$$
\left(\frac{2^{\mu} t^{\nu}}{\lambda e^{t}+1}\right)^{\alpha} e^{x t}=\sum_{n=0}^{\infty} F_{n}^{(\alpha)}(x ; \lambda ; \mu, \nu) \frac{t^{n}}{n !},(|t|<|\log (-\lambda)|)
$$

where

$$
F_{n}^{(\alpha)}(\lambda ; \mu, \nu)=F_{n}^{(\alpha)}(0 ; \lambda ; \mu, \nu)
$$

denote the so called Apostol type numbers of order $\alpha$.

So that by comparing equations (1.4), (1.5) and (1.6), we have

$$
\begin{gathered}
B_{n}^{(\alpha)}(x ; \lambda)=(-1)^{\alpha} F_{n}^{(\alpha)}(x ;-\lambda ; 0,1) \\
E_{n}^{(\alpha)}(x ; \lambda)=F_{n}^{(\alpha)}(x ; \lambda ; 1,0) \\
G_{n}^{(\alpha)}(x ; \lambda)=F_{n}^{(\alpha)}(x ; \lambda ; 1,1)
\end{gathered}
$$

Definition 1.3. Let $c>0$. The generalized 2-variable 1-parameter Hermite Kamp'e de Feriet polynomials $H_{n}(x, y, c)$ for nonnegative integer $\mathrm{n}$ are defined by

$$
c^{x t+y t^{2}}=\sum_{n=0}^{\infty} H_{n}(x, y, c) \frac{t^{n}}{n !}
$$

This is an extended 2-variable Hermite Kamp'e de Feriet polynomials $H_{n}(x, y)$ (see [3]) defined by

$$
e^{x t+y t^{2}}=\sum_{n=0}^{\infty} H_{n}(x, y) \frac{t^{n}}{n !}
$$


Note that

$$
H_{n}(x, y, e)=H_{n}(x, y)
$$

In order to collect the powers of $t$ we expand the left hand side of (1.15) to get the representation

$$
H_{n}(x, y, c)=\sum_{j=0}^{\left[\frac{n}{2}\right]}\left(\begin{array}{c}
n \\
j
\end{array}\right)(\ln c)^{n-j} x^{n-2 j} y^{j}
$$

In this paper, we first give definitions of the generalized Apostol type polynomials $F_{n}^{(\alpha)}(x ; \lambda ; u, \nu, c)$. which generalize the concepts stated above and then research their basic properties and relationships with Apostol type polynomials $F_{n}^{(\alpha)}(x ; \lambda ; u, \nu, c)$ and generalized Apostol type Hermite-Based polynomials ${ }_{H} F_{n}^{(\alpha)}(x, y ; \lambda ; u, \nu, c)$ of Lu et al [2]. Some implicit summation formulae and general symmetry identities are derived by using different analytical means and applying generating functions. These results extend some known summations and identities of generalized Apostol type Hermite-Bernoulli, Euler and Genocchi polynomials studied by Dattoli et al [6], Yang et al [22], Pathan[13], Zhang et al [25], Yang [10], Pathan and Khan [14].

\section{Definitions and Properties of the Generalized Apostol Type Hermite- Based Polynomials ${ }_{H} F_{n}^{(\alpha)}(x, y ; \lambda ; \mu, \nu, c)$}

In this section, we present some further definitions and properties for the generalized Apostol type Hermite-based polynomials ${ }_{H} F_{n}^{(\alpha)}(x, y ; \lambda ; \mu, \nu, c)$ :

Definition 2.1. The generalized Apostol type polynomials $F_{n}^{(\alpha)}(x, \lambda ; \mu, \nu, c)$ $\left(\alpha \epsilon N_{0}, \mu, \nu \epsilon C\right)$ for nonnegative integer $\mathrm{n}$ are defined by

$$
\left(\frac{2^{\mu} t^{\nu}}{\lambda c^{t}+1}\right)^{\alpha} c^{x t}=\sum_{n=0}^{\infty} F_{n}^{(\alpha)}(x ; \lambda ; \mu, \nu, c) \frac{t^{n}}{n !},(|t|<|\log (-\lambda)|)
$$

Definition 2.2. The generalized Apostol type Hermite-based polynomials ${ }_{H} F_{n}^{(\alpha)}$ $(x, y ; \lambda ; \mu, \nu, c)\left(\alpha \epsilon N_{0}, \mu, \nu \epsilon C\right)$ for nonnegative integer $\mathrm{n}$ are defined by

$$
\left(\frac{2^{\mu} t^{\nu}}{\lambda c^{t}+1}\right)^{\alpha} c^{x t+y t^{2}}=\sum_{n=0}^{\infty} H_{n} F_{n}^{(\alpha)}(x, y ; \lambda ; \mu, \nu, c) \frac{t^{n}}{n !},(|t|<|\log (-\lambda)|)
$$

For $\alpha=1$, we obtain from (2.2) the generating function

$$
\left(\frac{2^{\mu} t^{\nu}}{\lambda c^{t}+1}\right) c^{x t+y t^{2}}=\sum_{n=0}^{\infty}{ }_{H} F_{n}(x, y ; \lambda ; \mu, \nu, c) \frac{t^{n}}{n !},(|t|<|\log (-\lambda)|)
$$


whereas for $x=0$ gives

$$
F_{n}^{(\alpha)}(0, y ; \lambda, \mu, \nu, c)=\sum_{k=0}^{\left[\frac{n}{2}\right]} \frac{n !}{k !(n-2 k) !}(\ln c)^{k} F_{n-2 k}^{(\alpha)}(\lambda ; \mu, \nu) y^{k}
$$

Another special case of (2.2) for $y=0$ and $c=e$ leads to the extension of the generalized Apostol type polynomials $F_{n}^{(\alpha)}(x, \lambda ; \mu, \nu)$ for nonnegative integer $\mathrm{n}$ defined by (1.10) in the form

Further setting $c=e$ in $(2.2)$, we get

Definition 2.3. The generalized Apostol type Hermite-based polynomials ${ }_{H} F_{n}^{(\alpha)}$ $(x, y ; \lambda, \mu, \nu, e)\left(\alpha \epsilon N_{0}, \mu, \nu \epsilon C\right)$ for nonnegative integer $\mathrm{n}$ are defined by

$$
\left(\frac{2^{\mu} t^{\nu}}{\lambda e^{t}+1}\right)^{\alpha} e^{x t+y t^{2}}=\sum_{n=0}^{\infty}{ }_{H} F_{n}^{(\alpha)}(x, y ; \lambda ; \mu, \nu, e) \frac{t^{n}}{n !},(|t|<|\log (-\lambda)|)
$$

The generalized Apostol type Hermite-based polynomials ${ }_{H} F_{n}^{(\alpha)}(x, y ; \lambda ; \mu, \nu, c)$ defined by (2.2) have the following properties which are stated as theorems below.

Theorem 2.1. For any integral $n \geq 1, x, y \in R, \lambda \epsilon C$ and $\alpha \epsilon N$. The following relation for the generalized Apostol type polynomials ${ }_{H} F_{n}^{(\alpha)}(x, y ; \lambda ; \mu, \nu, c)$ holds true:

$$
\begin{aligned}
{ }_{H} F_{n}^{(\alpha)}(x, y ; \lambda ; \mu, \nu, e) & ={ }_{H} F_{n}^{(\alpha)}(x, y ; \lambda ; \mu, \nu),(-1)^{\alpha}{ }_{H} F_{n}^{(\alpha)}(x, y ;-\lambda ; 0,1, e) \\
& ={ }_{H} B_{n}^{(\alpha)}(x, y ; \lambda),
\end{aligned}
$$

$$
\begin{gathered}
{ }_{H} F_{n}^{(\alpha)}(x, y ; \lambda ; 1,0, e)={ }_{H} E_{n}^{(\alpha)}(x, y ; \lambda),{ }_{H} F_{n}^{(\alpha)}(x, y ; \lambda ; 1,1, e)={ }_{H} G_{n}^{(\alpha)}(x, y ; \lambda) \\
{ }_{H} F_{n}^{(\alpha+\beta)}(x+u, y+z ; \lambda ; \mu, \nu, c) \\
=\sum_{m=0}^{n}\left(\begin{array}{c}
n \\
m
\end{array}\right){ }_{H} F_{m}^{(\beta)}(u, z ; \lambda ; \mu, \nu, c)_{H} F_{n-m}^{(\alpha)}(x, y ; \lambda ; \mu, \nu, c) \\
{ }_{H} F_{n}^{(\alpha)}(x+z, y ; \lambda ; \mu, \nu, c)=\sum_{m=0}^{n}\left(\begin{array}{c}
n \\
m
\end{array}\right) F_{n-m}^{(\alpha)}(z ; \lambda ; \mu, \nu, c) H_{m}(x, y ; c)
\end{gathered}
$$

Proof. The formulas in (2.6) are obvious. Applying Definition (2.2), we have

$$
\sum_{n=0}^{\infty}{ }_{H} F_{n}^{(\alpha+\beta)}(x+u, y+z ; \lambda ; \mu, \nu, c) \frac{t^{n}}{n !}
$$




$$
\begin{aligned}
& =\sum_{n=0}^{\infty}{ }_{H} F_{n}^{(\alpha)}(x, y ; \lambda ; \mu, \nu, c) \frac{t^{n}}{n !} \sum_{m=0}^{\infty}{ }_{H} F_{m}^{(\beta)}(u, z ; \lambda ; \mu, \nu, c) \frac{t^{m}}{m !} \\
= & \sum_{n=0}^{\infty} \sum_{m=0}^{n}{ }_{H} F_{m}^{(\beta)}(u, z ; \lambda ; \mu, \nu, c)_{H} F_{n-m}^{(\alpha)}(x, y ; \lambda ; \mu, \nu, c) \frac{t^{n}}{(n-m) !}
\end{aligned}
$$

Now equating the coefficients of the like powers of $t$ in the above equation, we get the result (2.7). Again by Definition (2.2), we have

$$
\left(\frac{2^{\mu} t^{\nu}}{\lambda c^{t}+1}\right)^{\alpha} c^{(x+z) t+y t^{2}}=\sum_{n=0}^{\infty}{ }_{H} F_{n}^{(\alpha)}(x+z, y ; \lambda ; \mu, \nu, c) \frac{t^{n}}{n !}
$$

which can be written as

$$
\left(\frac{2^{\mu} t^{\nu}}{\lambda c^{t}+1}\right)^{\alpha} c^{z t} c^{x t+y t^{2}}=\sum_{n=0}^{\infty} F_{n}^{(\alpha)}(z ; \lambda ; \mu, \nu, c) \frac{t^{n}}{n !} \sum_{m=0}^{\infty} H_{m}(x, y ; c) \frac{t^{n}}{n !}
$$

Replacing $\mathrm{n}$ by $\mathrm{n}-\mathrm{m}$ in (2.10), comparing with (2.9) and equating their coefficients of $t^{n}$ leads to formula (2.8).

\section{Implicit Summation Formulae Involving Generalized Apostol Type Hermite-Based Polynomials}

For the derivation of implicit formulae involving generalized Apostol type polynomials $F_{n}^{(\alpha)}(x, y ; \lambda ; \mu, \nu, c)$ and generalized Apostol type Hermite-Based polynomials ${ }_{H} F_{n}^{(\alpha)}(x, y ; \lambda ; \mu, \nu, c)$ the same considerations as developed for the ordinary Hermite and related polynomials in Khan et al [21] and Hermite-Bernoulli polynomials in Pathan [13], Pathan and Khan [14] holds as well. First we prove the following results involving generalized Apostol type Hermite-Based polynomials ${ }_{H} F_{n}^{(\alpha)}(x, y ; \lambda ; \mu, \nu, c)$.

Theorem 3.1. For any integral $n \geq 1, x, y \in R, \lambda \epsilon C$ and $\alpha \epsilon N$. Then the following implicit summation formulae for generalized Apostol type Hermit-based polynomials ${ }_{H} F_{n}^{(\alpha)}(x, y ; \lambda ; \mu, \nu, c)$ holds true:

${ }_{H} F_{k+l}^{(\alpha)}(z, y ; \lambda ; \mu, \nu, c)=\sum_{n, m=0}^{k, l}\left(\begin{array}{l}l \\ m\end{array}\right)\left(\begin{array}{l}k \\ n\end{array}\right)(z-x)^{n+m}{ }_{H} F_{k+l-n-m}^{(\alpha)}(x, y ; \lambda ; \mu, \nu, c)$

Proof. We replace t by $t+w$ and rewrite the generating function (2.2) as

$$
\left(\frac{2^{\mu}(t+w)^{\nu}}{\lambda c^{t+w}+1}\right)^{\alpha} c^{y(t+w)^{2}}=c^{-x(t+w)} \sum_{k, l=0}^{\infty}{ }_{H} F_{k+l}^{(\alpha)}(x, y ; \lambda ; \mu, \nu, c) \frac{t^{k}}{k !} \frac{w^{l}}{l !}
$$


Replacing $\mathrm{x}$ by $\mathrm{z}$ in the above equation and equating the resulting equation to the above equation, we get

$$
c^{(z-x)(t+w)} \sum_{k, l=0}^{\infty}{ }_{H} F_{k+l}^{(\alpha)}(x, y ; \lambda ; \mu, \nu, c) \frac{t^{k}}{k !} \frac{w^{l}}{l !}=\sum_{k, l=0}^{\infty}{ }_{H} F_{k+l}^{(\alpha)}(z, y \lambda ; \mu, \nu, c) \frac{t^{k}}{k !} \frac{w^{l}}{l !}
$$

On expanding exponential function, (3.3) gives

$$
\begin{gathered}
\sum_{N=0}^{\infty} \frac{[(z-x)(t+w)]^{N}}{N !} \sum_{k, l=0}^{\infty}{ }_{H} F_{k+l}^{(\alpha)}(x, y ; \lambda ; \mu, \nu, c) \frac{t^{k}}{k !} \frac{w^{l}}{l !} \\
=\sum_{k, l=0}^{\infty}{ }_{H} F_{k+l}^{(\alpha)}(z, y ; \lambda ; \mu, \nu, c) \frac{t^{k}}{k !} \frac{w^{l}}{l !}
\end{gathered}
$$

which on using formula [7,p.52(2)]

$$
\sum_{N=0}^{\infty} f(N) \frac{(x+y)^{N}}{N !}=\sum_{n, m=0}^{\infty} f(n+m) \frac{x^{n}}{n !} \frac{y^{m}}{m !}
$$

in the left hand side becomes

$$
\begin{gathered}
\sum_{n, m=0}^{\infty} \frac{(z-x)^{n+m} t^{n} w^{m}}{n ! m !} \sum_{k, l=0}^{\infty}{ }_{H} F_{k+l}^{(\alpha)}(x, y ; \lambda ; \mu, \nu, c) \frac{t^{k}}{k !} \frac{w^{l}}{l !} \\
=\sum_{k, l=0}^{\infty}{ }_{H} F_{k+l}^{(\alpha)}(z, y ; \lambda ; \mu, \nu, c) \frac{t^{k}}{k !} \frac{w^{l}}{l !}
\end{gathered}
$$

Now replacing $\mathrm{k}$ by k-n, 1 by l-m in the left hand side of (3.6), we get

$$
\begin{gathered}
\sum_{n, m=0}^{\infty} \sum_{k, l=0}^{\infty} \frac{(z-x)^{n+m}}{n ! m !}{ }_{H} F_{k+l-n-m}^{(\alpha)}(x, y ; \lambda ; \mu, \nu, c) \frac{t^{k}}{(k-n) !} \frac{w^{l}}{(l-m) !} \\
=\sum_{k, l=0}^{\infty} H_{k+l}^{(\alpha)}(z, y ; \lambda ; \mu, \nu, c) \frac{t^{k}}{k !} \frac{w^{l}}{l !}
\end{gathered}
$$

Finally on equating the coefficients of the like powers of $t$ and $u$ in the above equation, we get the required result.

By setting $\lambda=-\lambda, \mu=0$ and $\nu=1$ in Theorem (3.1) and then multiplying $(-1)^{\alpha}$ on both sides of the result, we have the following corollary 
Corollary 1. The following implicit summation formula for Apostol HermiteBernoulli polynomials ${ }_{H} B_{n}^{(\alpha)}(x, y ; \lambda ; c)$ holds true:

$$
{ }_{H} B_{k+l}^{(\alpha)}(z, y ; \lambda ; c)=\sum_{n, m=0}^{k, l}\left(\begin{array}{l}
l \\
m
\end{array}\right)\left(\begin{array}{l}
k \\
n
\end{array}\right)(z-x)^{n+m}{ }_{H} B_{k+l-n-m}^{(\alpha)}(x, y ; \lambda ; c)
$$

Remark. For $\lambda=1, c=e$ in (3.8), the result reduces to known result of Pathan and Khan [14, Theorem 3.1].

By setting $\mu=1$ and $\nu=0$ in Theorem (3.1), we have the following corollary

Corollary 2. The following implicit summation formula for Apostol Hermite-Euler polynomials ${ }_{H} E_{n}^{(\alpha)}(x, y ; \lambda ; c)$ holds true:

$$
{ }_{H} E_{k+l}^{(\alpha)}(z, y ; \lambda ; c)=\sum_{n, m=0}^{k, l}\left(\begin{array}{l}
l \\
m
\end{array}\right)\left(\begin{array}{l}
k \\
n
\end{array}\right)(z-x)^{n+m}{ }_{H} E_{k+l-n-m}^{(\alpha)}(x, y ; \lambda ; c)
$$

By setting $\mu=1$ and $\nu=1$ in Theorem (3.1), we have the following corollary

Corollary 3. The following implicit summation formula for Apostol HermiteGenocchi polynomials ${ }_{H} G_{n}^{(\alpha)}(x, y ; \lambda ; c)$ holds true:

$$
{ }_{H} G_{k+l}^{(\alpha)}(z, y ; \lambda ; c)=\sum_{n, m=0}^{k, l}\left(\begin{array}{l}
l \\
m
\end{array}\right)\left(\begin{array}{l}
k \\
n
\end{array}\right)(z-x)^{n+m}{ }_{H} G_{k+l-n-m}^{(\alpha)}(x, y ; \lambda ; c)
$$

Theorem 3.2. For any integral $n \geq 1, x, y \in R$ and $\alpha \in N$. Then

$$
{ }_{H} F_{n}^{(\alpha)}(x, y ; \lambda ; \mu, \nu, c)=\sum_{m=0}^{n}\left(\begin{array}{l}
n \\
m
\end{array}\right) F_{n-m}^{(\alpha)}(\lambda ; \mu, \nu, c) H_{m}(x, y, c)
$$

Proof. By the definition of generalized Apostol type polynomials and the definition (1.10), we have

$$
\begin{aligned}
& \left(\frac{2^{\mu} t^{\nu}}{\lambda c^{t}+1}\right)^{\alpha} c^{x t+y t^{2}}=\sum_{n=0}^{\infty}{ }_{H} F_{n}^{(\alpha)}(x, y ; \lambda ; \mu, \nu, c) \frac{t^{n}}{n !} \\
& =\left(\sum_{n=0}^{\infty} F_{n}^{(\alpha)}(\lambda ; \mu, \nu, c) \frac{t^{n}}{n !}\right)\left(\sum_{m=0}^{\infty} H_{m}(x, y ; c) \frac{t^{m}}{m !}\right)
\end{aligned}
$$

Now replacing $\mathrm{n}$ by $\mathrm{n}-\mathrm{m}$ and comparing the coefficients of $t^{n}$, we get the result (3.11).

Remark. For $c=e,(3.11)$ yields

$$
{ }_{H} F_{n}^{(\alpha)}(x, y ; \lambda ; \mu, \nu, e)=\sum_{m=0}^{n}\left(\begin{array}{l}
n \\
m
\end{array}\right) F_{n-m}^{\alpha}(\lambda ; \mu, \nu) H_{m}(x, y)
$$


By setting $\lambda=-\lambda, \mu=0$ and $\nu=1$ in Theorem (3.2) and then multiplying $(-1)^{\alpha}$ on both sides of the result, we have the following corollary

Corollary 1. For any integral $n \geq 1, x, y \in R$ and $\alpha \in N$. Then

$$
{ }_{H} B_{n}^{(\alpha)}(x, y ; \lambda ; c)=\sum_{m=0}^{n}\left(\begin{array}{l}
n \\
m
\end{array}\right) B_{n-m}^{(\alpha)}(\lambda ; c) H_{m}(x, y, c)
$$

By setting $\mu=1$ and $\nu=0$ in Theorem (3.2), we have the following corollary

Corollary 2. For any integral $n \geq 1, x, y \in R$ and $\alpha \in N$. Then

$$
{ }_{H} E_{n}^{(\alpha)}(x, y ; \lambda ; c)=\sum_{m=0}^{n}\left(\begin{array}{l}
n \\
m
\end{array}\right) E_{n-m}^{(\alpha)}(\lambda ; c) H_{m}(x, y, c)
$$

By setting $\mu=1$ and $\nu=1$ in Theorem (3.2), we have the following corollary

Corollary 3. For any integral $n \geq 1, x, y \in R$ and $\alpha \in N$. Then

$$
{ }_{H} G_{n}^{(\alpha)}(x, y ; \lambda ; c)=\sum_{m=0}^{n}\left(\begin{array}{l}
n \\
m
\end{array}\right) G_{n-m}^{(\alpha)}(\lambda ; c) H_{m}(x, y ; c)
$$

Theorem 3.3. For any integral $n \geq 1, x, y \in R$ and $\alpha \in N$. Then

$$
{ }_{H} F_{n}^{(\alpha)}(x, y ; \lambda ; \mu, \nu, c)=\sum_{k=0}^{n-2 j} \sum_{j=0}^{\left[\frac{n}{2}\right]}\left(\begin{array}{l}
n-2 j \\
k
\end{array}\right)(\ln c)^{n-k-j} x^{n-k-2 j} y^{j} F_{k}^{(\alpha)}(\lambda ; \mu, \nu, c)
$$

Proof. Applying the definition (2.2) to the term $\left(\frac{2^{\mu} t^{\nu}}{\lambda c^{t}+1}\right)^{\alpha}$ and expanding the exponential function $c^{x t+y t^{2}}$ at $t=0$ yields

$$
\begin{aligned}
& \left(\frac{2^{\mu} t^{\nu}}{\lambda c^{t}+1}\right)^{\alpha} c^{x t+y t^{2}} \\
& =\left(\sum_{k=0}^{\infty} F_{k}^{(\alpha)}(\lambda ; \mu, \nu, c) \frac{t^{k}}{k !}\right)\left(\sum_{n=0}^{\infty} x^{n}(\ln c)^{n} \frac{t^{n}}{n !}\right)\left(\sum_{j=0}^{\infty} y^{j}(\ln c)^{j} \frac{t^{2 j}}{j !}\right) \\
& =\sum_{n=0}^{\infty}\left(\sum_{k=0}^{n}\left(\begin{array}{c}
n \\
k
\end{array}\right)(\ln c)^{n-k} F_{k}^{(\alpha)}(\lambda ; \mu, \nu, c) x^{n-k}\right) \frac{t^{n}}{n !}\left(\sum_{j=0}^{\infty} y^{j}(\ln c)^{j} \frac{t^{2 j}}{j !}\right)
\end{aligned}
$$

Replacing $\mathrm{n}$ by $\mathrm{n}-2 \mathrm{j}$, we have

$$
\sum_{n=0}^{\infty}{ }_{H} F_{n}^{(\alpha)}(x, y ; \lambda ; \mu, \nu, c) \frac{t^{n}}{n !}
$$




$$
=\sum_{n=0}^{\infty}\left(\sum_{k=0}^{n-2 j} \sum_{j=0}^{\left[\frac{n}{2}\right]}\left(\begin{array}{l}
n-2 j \\
k
\end{array}\right)(\ln c)^{n-k-j} x^{n-k-2 j} y^{j} F_{k}^{(\alpha)}(\lambda ; \mu, \nu, c)\right) t^{n}
$$

Combining (3.16) and (2.2) and equating their coefficients of $t^{n}$ produce the formula (3.15).

By setting $\lambda=-\lambda, \mu=0$ and $\nu=1$ in Theorem (3.3) and then multiplying $(-1)^{\alpha}$ on both sides of the result, we have the following corollary

Corollary 1. For any integral $n \geq 1, x, y \in R$ and $\alpha \epsilon N$. Then

$$
{ }_{H} B_{n}^{(\alpha)}(x, y ; \lambda ; c)=\sum_{k=0}^{n-2 j} \sum_{j=0}^{\left[\frac{n}{2}\right]}\left(\begin{array}{l}
n-2 j \\
k
\end{array}\right)(\ln c)^{n-k-j} x^{n-k-2 j} y^{j} B_{k}^{(\alpha)}(\lambda ; c)
$$

By setting $\mu=1$ and $\nu=0$ in Theorem (3.3), we have the following corollary

Corollary 2. For any integral $n \geq 1, x, y \in R$ and $\alpha \in N$. Then

$$
{ }_{H} E_{n}^{(\alpha)}(x, y ; \lambda ; c)=\sum_{k=0}^{n-2 j} \sum_{j=0}^{\left[\frac{n}{2}\right]}\left(\begin{array}{l}
n-2 j \\
k
\end{array}\right)(\ln c)^{n-k-j} x^{n-k-2 j} y^{j} E_{k}^{(\alpha)}(\lambda ; c)
$$

By setting $\mu=1$ and $\nu=1$ in Theorem (3.3), we have the following corollary

Corollary 3. For any integral $n \geq 1, x, y \in R$ and $\alpha \in N$. Then

$$
{ }_{H} G_{n}^{(\alpha)}(x, y ; \lambda ; c)=\sum_{k=0}^{n-2 j} \sum_{j=0}^{\left[\frac{n}{2}\right]}\left(\begin{array}{l}
n-2 j \\
k
\end{array}\right)(\ln c)^{n-k-j} x^{n-k-2 j} y^{j} G_{k}^{(\alpha)}(\lambda ; c)
$$

Theorem 3.4. For any integral $n \geq 1, x, y \in R$ and $\alpha \in N$. Then

$$
{ }_{H} F_{n}^{(\alpha)}(x+1, y ; \lambda ; \mu, \nu, c)=\sum_{j=0}^{\left[\frac{n}{2}\right]} \sum_{k=0}^{n-2 j}\left(\begin{array}{l}
n-2 j \\
k
\end{array}\right)(\ln c)^{n-k-j} y^{j} F_{k}^{(\alpha)}(x ; \lambda ; \mu, \nu, c)
$$

Proof. By the definition of generalized Apostol type Hermite-based polynomials, we have

$$
\begin{gathered}
\left(\frac{2^{\mu} t^{\nu}}{\lambda c^{t}+1}\right)^{\alpha} c^{(x+1) t+y t^{2}}=\sum_{n=0}^{\infty}{ }_{H} F_{n}^{(\alpha)}(x+1, y ; \lambda ; \mu, \nu, c) \frac{t^{n}}{n !} \\
=\left(\sum_{k=0}^{\infty} F_{k}^{(\alpha)}(x ; \lambda ; \mu, \nu, c) \frac{t^{k}}{k !}\right)\left(\sum_{n=0}^{\infty}(\ln c)^{n} \frac{t^{n}}{n !}\right)\left(\sum_{j=0}^{\infty} y^{j}(\ln c)^{j} \frac{t^{2 j}}{j !}\right)
\end{gathered}
$$




$$
\begin{aligned}
& =\sum_{n=0}^{\infty} \sum_{k=0}^{n}\left(\begin{array}{c}
n \\
k
\end{array}\right)(\ln c)^{n-k} F_{k}^{(\alpha)}(x ; \lambda ; \mu, \nu, c) \frac{t^{n}}{n !}\left(\sum_{j=0}^{\infty} y^{j}(\ln c)^{j} \frac{t^{2 j}}{j !}\right) \\
& =\sum_{n=0}^{\infty} \sum_{j=0}^{\infty} \sum_{k=0}^{n}\left(\begin{array}{c}
n \\
k
\end{array}\right)(\ln c)^{n-k+j} y^{j} F_{k}^{(\alpha)}(x ; \lambda ; \mu, \nu, c) \frac{t^{n+2 j}}{n ! j !}
\end{aligned}
$$

Replacing $\mathrm{n}$ by $\mathrm{n}-2 \mathrm{j}$, we have

$$
\begin{gathered}
\sum_{n=0}^{\infty}{ }_{H} F_{n}^{(\alpha)}(x+1, y ; \lambda ; \mu, \nu, c) \frac{t^{n}}{n !} \\
=\sum_{n=0}^{\infty} \sum_{j=0}^{\left[\frac{n}{2}\right]} \sum_{k=0}^{n-2 j}\left(\begin{array}{l}
n-2 j \\
k
\end{array}\right)(\ln c)^{n-k-j} y^{j} F_{k}^{(\alpha)}(x ; \lambda ; \mu, \nu, c) t^{n}
\end{gathered}
$$

Combining (3.21) and (3.23) and equating their coefficients of $t^{n}$ leads to formula (3.20).

By setting $\lambda=-\lambda, \mu=0$ and $\nu=1$ in Theorem (3.4) and then multiplying $(-1)^{\alpha}$ on both sides of the result, we have the following corollary

Corollary 1. For any integral $n \geq 1, x, y \in R$ and $\alpha \in N$. Then

$$
{ }_{H} B_{n}^{(\alpha)}(x+1, y ; \lambda ; c)=\sum_{j=0}^{\left[\frac{n}{2}\right]} \sum_{k=0}^{n-2 j}\left(\begin{array}{l}
n-2 j \\
k
\end{array}\right)(\ln c)^{n-k-j} y^{j} B_{k}^{(\alpha)}(x ; \lambda ; c)
$$

By setting $\mu=1$ and $\nu=0$ in Theorem (3.4), we have the following corollary

Corollary 2. For any integral $n \geq 1, x, y \in R$ and $\alpha \in N$. Then

$$
{ }_{H} E_{n}^{(\alpha)}(x+1, y ; \lambda ; c)=\sum_{j=0}^{\left[\frac{n}{2}\right]} \sum_{k=0}^{n-2 j}\left(\begin{array}{l}
n-2 j \\
k
\end{array}\right)(\ln c)^{n-k-j} y^{j} E_{k}^{(\alpha)}(x ; \lambda ; c)
$$

By setting $\mu=1$ and $\nu=1$ in Theorem (3.4), we have the following corollary

Corollary 3. For any integral $n \geq 1, x, y \in R$ and $\alpha \epsilon N$. Then

$$
{ }_{H} G_{n}^{(\alpha)}(x+1, y ; \lambda ; c)=\sum_{j=0}^{\left[\frac{n}{2}\right]} \sum_{k=0}^{n-2 j}\left(\begin{array}{l}
n-2 j \\
k
\end{array}\right)(\ln c)^{n-k-j} y^{j} G_{k}^{(\alpha)}(x ; \lambda ; c)
$$

Theorem 3.5. For any integral $n \geq 1, x, y \in R$ and $\alpha \in N$. Then

$$
{ }_{H} F_{n}^{(\alpha)}(x, y ; \lambda ; \mu, \nu, e)=\sum_{m=0}^{n}\left(\begin{array}{l}
n \\
m
\end{array}\right) F_{n-m}^{(\alpha-1)}(\lambda ; \mu, \nu)_{H} F_{m}^{(\alpha)}(x, y ; \lambda ; \mu, \nu, e)
$$


Proof. By the definition of generalized Apostol type Hermite-based polynomials, we have

$$
\begin{gathered}
\frac{2^{\mu} t^{\nu}}{\lambda e^{t}+1}\left(\frac{2^{\mu} t^{\nu}}{\lambda e^{t}+1}\right)^{\alpha} e^{x t+y t^{2}}=\frac{2^{\mu} t^{\nu}}{\lambda e^{t}+1} \sum_{n=0}^{\infty}{ }_{H} F_{n}^{(\alpha)}(x, y ; \lambda ; \mu, \nu, e) \frac{t^{n}}{n !} \\
\left(\frac{2^{\mu} t^{\nu}}{\lambda e^{t}+1}\right)^{\alpha} e^{x t+y t^{2}}=\frac{2^{\mu} t^{\nu}}{\lambda e^{t}+1} \sum_{m=0}^{\infty}{ }_{H} F_{m}^{(\alpha)}(x, y ; \lambda ; \mu, \nu, e) \frac{t^{m}}{m !}
\end{gathered}
$$

Now replacing $\mathrm{n}$ by $\mathrm{n}-\mathrm{m}$ and equating the coefficients of $t^{n}$ leads to formula (3.27).

By setting $\lambda=-\lambda, \mu=0$ and $\nu=1$ in Theorem (3.5) and then multiplying $(-1)^{\alpha}$ on both sides of the result, we have the following corollary

Corollary 1. For any integral $n \geq 1, x, y \in R$ and $\alpha \in N$. Then

$$
{ }_{H} B_{n}^{(\alpha)}(x, y ; \lambda ; e)=\sum_{m=0}^{n}\left(\begin{array}{c}
n \\
m
\end{array}\right) B_{n-m}^{(\alpha-1)}(\lambda)_{H} B_{m}^{(\alpha)}(x, y ; \lambda ; e)
$$

By setting $\mu=1$ and $\nu=0$ in Theorem (3.5), we have the following corollary

Corollary 2. For any integral $n \geq 1, x, y \in R$ and $\alpha \in N$. Then

$$
{ }_{H} E_{n}^{(\alpha)}(x, y ; \lambda ; e)=\sum_{m=0}^{n}\left(\begin{array}{c}
n \\
m
\end{array}\right) E_{n-m}^{(\alpha-1)}(\lambda)_{H} E_{m}^{(\alpha)}(x, y ; \lambda ; e)
$$

By setting $\mu=1$ and $\nu=1$ in Theorem (3.5), we have the following corollary

Corollary 3. For any integral $n \geq 1, x, y \in R$ and $\alpha \epsilon N$. Then

$$
{ }_{H} G_{n}^{(\alpha)}(x, y ; \lambda ; e)=\sum_{m=0}^{n}\left(\begin{array}{c}
n \\
m
\end{array}\right) G_{n-m}^{(\alpha-1)}(\lambda)_{H} G_{m}^{(\alpha)}(x, y ; \lambda ; e)
$$

Theorem 3.6. For arbitrary real or complex parameter $\alpha$, the following implicit summation formula involving generalized Apostol type polynomials ${ }_{H} F_{n}^{(\alpha)}(x, y ; \lambda ; \mu, \nu, c)$ holds true:

$$
{ }_{H} F_{n}^{(\alpha)}(x+1, y ; \lambda ; \mu, \nu, c)=\sum_{k=0}^{n}\left(\begin{array}{c}
n \\
k
\end{array}\right)(\ln c)^{n-k}{ }_{H} F_{k}^{(\alpha)}(x, y ; \lambda ; \mu, \nu, c)
$$

Proof. By the definition of generalized Apostol type Hermite-based polynomials, we have

$$
\sum_{n=0}^{\infty}{ }_{H} F_{n}^{(\alpha)}(x+1, y ; \lambda ; \mu, \nu, c) \frac{t^{n}}{n !}-\sum_{n=0}^{\infty}{ }_{H} F_{n}^{(\alpha)}(x, y ; \lambda ; \mu, \nu, c) \frac{t^{n}}{n !}
$$




$$
\begin{gathered}
=\left(\frac{2^{\mu} t^{\nu}}{\lambda c^{t}+1}\right)^{\alpha} c^{x t+y t^{2}}\left(c^{t}-1\right) \\
=\left(\sum_{k=0}^{\infty}{ }_{H} F_{k}^{(\alpha)}(x, y ; \lambda ; \mu, \nu, c) \frac{t^{k}}{k !}\right)\left(\sum_{n=0}^{\infty}(\ln c)^{n} \frac{t^{n}}{n !}\right)-\sum_{n=0}^{\infty}{ }_{H} F_{n}^{(\alpha)}(x, y ; \lambda ; \mu, \nu, c) \frac{t^{n}}{n !} \\
=\sum_{n=0}^{\infty} \sum_{k=0}^{n}(\ln c)^{n-k}{ }_{H} F_{k}^{(\alpha)}(x, y ; \lambda ; \mu, \nu, c) \frac{t^{n}}{(n-k) !}-\sum_{n=0}^{\infty}{ }_{H} F_{n}^{(\alpha)}(x, y ; \lambda ; \mu, \nu, c) \frac{t^{n}}{n !}
\end{gathered}
$$

Finally, equating the coefficients of the like powers of $t^{n}$, we get (3.31).

By setting $\lambda=-\lambda, \mu=0$ and $\nu=1$ in Theorem (3.6) and then multiplying $(-1)^{\alpha}$ on both sides of the result, we have the following corollary

Corollary 1. For any integral $n \geq 1, x, y \in R$ and $\alpha \in N$. Then

$$
{ }_{H} B_{n}^{(\alpha)}(x+1, y ; \lambda ; c)=\sum_{k=0}^{n}\left(\begin{array}{c}
n \\
k
\end{array}\right)(\ln c)^{n-k}{ }_{H} B_{k}^{(\alpha)}(x, y ; \lambda ; c)
$$

By setting $\mu=1$ and $\nu=0$ in Theorem (3.6), we have the following corollary

Corollary 2. For any integral $n \geq 1, x, y \in R$ and $\alpha \in N$. Then

$$
{ }_{H} E_{n}^{(\alpha)}(x+1, y ; \lambda ; c)=\sum_{k=0}^{n}\left(\begin{array}{c}
n \\
k
\end{array}\right)(\ln c)^{n-k}{ }_{H} E_{k}^{(\alpha)}(x, y ; \lambda ; c)
$$

By setting $\mu=1$ and $\nu=1$ in Theorem (3.6), we have the following corollary

Corollary 3. For any integral $n \geq 1, x, y \in R$ and $\alpha \in N$. Then

$$
{ }_{H} G_{n}^{(\alpha)}(x+1, y ; \lambda ; c)=\sum_{k=0}^{n}\left(\begin{array}{c}
n \\
k
\end{array}\right)(\ln c)^{n-k}{ }_{H} G_{k}^{(\alpha)}(x, y ; \lambda ; c)
$$

\section{Identities}

In this section, we give general symmetry identities for the generalized Apostol type polynomials $F_{n}^{(\alpha)}(x ; \lambda ; \mu, \nu, c)$ and the generalized Apostol type Hermite-based

polynomials ${ }_{H} F_{n}^{(\alpha)}(x, y ; \lambda ; \mu, \nu, c)$ by applying the generating functions (2.1) and (2.2). The results extend some known identities of Zhang et al [25], Yang et al [22], Lu et al [2], Pathan [13], Yang [10] and Pathan and Khan [14]. Throughout this section $\alpha$ will taken as an arbitrary real or complex parameter.

Theorem 4.1. For any integral $n \geq 1, x, y \in R$ and $\alpha \in N$. Then the following identity holds true:

$$
\sum_{k=0}^{n}\left(\begin{array}{c}
n \\
k
\end{array}\right) b^{k} a^{n-k}{ }_{H} F_{n-k}^{(\alpha)}\left(b x, b^{2} y ; \lambda ; \mu, \nu, c\right)_{H} F_{k}^{(\alpha)}\left(a x, a^{2} y ; \lambda ; \mu, \nu, c\right)
$$




$$
=\sum_{k=0}^{n}\left(\begin{array}{c}
n \\
k
\end{array}\right) a^{k} b^{n-k}{ }_{H} F_{n-k}^{(\alpha)}\left(a x, a^{2} y ; \lambda ; \mu, \nu, c\right)_{H} F_{k}^{(\alpha)}\left(b x, b^{2} y ; \lambda ; \mu, \nu, c\right)
$$

Proof. Start with

$$
g(t)=\left(\frac{(a b)^{\nu} 2^{2 \mu} t^{2 \nu}}{\left(\lambda c^{a t}+1\right)\left(\lambda c^{b t}+1\right)}\right)^{\alpha} c^{a b x t+a^{2} b^{2} y t^{2}}
$$

Then the expression for $\mathrm{g}(\mathrm{t})$ is symmetric in $\mathrm{a}$ and $\mathrm{b}$ and we can expand $\mathrm{g}(\mathrm{t})$ into series in two ways to obtain

$$
\begin{aligned}
& g(t)=\sum_{n=0}^{\infty}{ }_{H} F_{n}^{(\alpha)}\left(b x, b^{2} y ; \lambda ; \mu, \nu, c\right) \frac{(a t)^{n}}{n !} \sum_{k=0}^{\infty}{ }_{H} F_{k}^{(\alpha)}\left(a x, a^{2} y ; \lambda ; \mu, \nu, c\right) \frac{(b t)^{k}}{k !} \\
& =\sum_{n=0}^{\infty} \sum_{k=0}^{n}{ }_{H} F_{n-k}^{(\alpha)}\left(b x, b^{2} y ; \lambda ; \mu, \nu, c\right) a^{n-k} b^{k}{ }_{H} F_{k}^{(\alpha)}\left(a x, a^{2} y ; \lambda ; \mu, \nu, c\right) \frac{t^{n}}{(n-k) !}
\end{aligned}
$$

On the similar lines we can show that

$$
\begin{aligned}
& g(t)=\sum_{n=0}^{\infty}{ }_{H} F_{n}^{(\alpha)}\left(a x, a^{2} y ; \lambda ; \mu . \nu, c\right) \frac{(b t)^{n}}{n !} \sum_{k=0}^{\infty}{ }_{H} F_{k}^{(\alpha)}\left(b x, b^{2} y ; \lambda ; \mu, \nu, c\right) \frac{(a t)^{k}}{k !} \\
& =\sum_{n=0}^{\infty} \sum_{k=0}^{n}{ }_{H} F_{n-k}^{(\alpha)}\left(a x, a^{2} y ; \lambda ; \mu, \nu, c\right) a^{k} b^{n-k}{ }_{H} F_{k}^{(\alpha)}\left(b x, b^{2} y ; \lambda ; \mu, \nu, c\right) \frac{t^{n}}{(n-k) !}
\end{aligned}
$$

By comparing the coefficients of $t^{n}$ on the right hand sides of the last two equations we arrive the desired result.

By setting $\lambda=-\lambda, \mu=0$ and $\nu=1$ in Theorem (4.1) and then multiplying $(-1)^{\alpha}$ on both sides of the result, we have the following corollary

Corollary 1. For any integral $n \geq 1, x, y \in R$ and $\alpha \in N$. Then

$$
\begin{aligned}
& \sum_{k=0}^{n}\left(\begin{array}{c}
n \\
k
\end{array}\right) b^{k} a^{n-k}{ }_{H} B_{n-k}^{(\alpha)}\left(b x, b^{2} y ; \lambda ; c\right)_{H} B_{k}^{(\alpha)}\left(a x, a^{2} y ; \lambda ; c\right) \\
= & \sum_{k=0}^{n}\left(\begin{array}{c}
n \\
k
\end{array}\right) a^{k} b^{n-k}{ }_{H} B_{n-k}^{(\alpha)}\left(a x, a^{2} y ; \lambda ; c\right)_{H} B_{k}^{(\alpha)}\left(b x, b^{2} y ; \lambda ; c\right)
\end{aligned}
$$

Remark. For $\lambda=1, c=e$ in (4.3), the result reduces to known result of Pathan and Khan [14, Theorem 4.1].

By setting $\mu=1$ and $\nu=0$ in Theorem (4.1), we have the following corollary 
Corollary 2. For any integral $n \geq 1, x, y \in R$ and $\alpha \in N$. Then

$$
\begin{aligned}
& \sum_{k=0}^{n}\left(\begin{array}{c}
n \\
k
\end{array}\right) b^{k} a^{n-k} E_{n-k}^{(\alpha)}\left(b x, b^{2} y ; \lambda ; c\right)_{H} E_{k}^{(\alpha)}\left(a x, a^{2} y ; \lambda ; c\right) \\
= & \sum_{k=0}^{n}\left(\begin{array}{c}
n \\
k
\end{array}\right) a^{k} b^{n-k} E_{n-k}^{(\alpha)}\left(a x, a^{2} y ; \lambda ; c\right)_{H} E_{k}^{(\alpha)}\left(b x, b^{2} y ; \lambda ; c\right)
\end{aligned}
$$

By setting $\mu=1$ and $\nu=1$ in Theorem (4.1), we have the following corollary

Corollary 3. For any integral $n \geq 1, x, y \in R$ and $\alpha \epsilon N$. Then

$$
\begin{aligned}
& \sum_{k=0}^{n}\left(\begin{array}{c}
n \\
k
\end{array}\right) b^{k} a^{n-k}{ }_{H} G_{n-k}^{(\alpha)}\left(b x, b^{2} y ; \lambda ; c\right)_{H} G_{k}^{(\alpha)}\left(a x, a^{2} y ; \lambda ; c\right) \\
= & \sum_{k=0}^{n}\left(\begin{array}{c}
n \\
k
\end{array}\right) a^{k} b^{n-k} G_{n-k}^{(\alpha)}\left(a x, a^{2} y ; \lambda ; c\right)_{H} G_{k}^{(\alpha)}\left(b x, b^{2} y ; \lambda ; c\right)
\end{aligned}
$$

Theorem 4.2. For any integral $n \geq 1, x, y \in R$ and $\alpha \in N$. Then the following identity holds true:

$$
\begin{gathered}
\sum_{k=0}^{n}\left(\begin{array}{c}
n \\
k
\end{array}\right) \sum_{i=0}^{a-1} \sum_{j=0}^{b-1}(-\lambda)^{i+j} a^{n-k} b_{H}^{k} F_{n-k}^{(\alpha)} \\
\left(b x+\frac{b}{a} i+j, b^{2} z ; \lambda ; \mu, \nu, c\right) F_{k}^{(\alpha)}(a y ; \lambda ; \mu, \nu, c) \\
=\sum_{k=0}^{n}\left(\begin{array}{c}
n \\
k
\end{array}\right) \sum_{i=0}^{b-1} \sum_{j=0}^{a-1}(-\lambda)^{i+j} a^{k} b^{n-k}{ }_{H} F_{n-k}^{(\alpha)} \\
\left(a x+\frac{a}{b} i+j, a^{2} z ; \lambda ; \mu, \nu, c\right) F_{k}^{(\alpha)}(b y ; \lambda ; \mu, \nu, c)
\end{gathered}
$$

Proof. Let

$$
\begin{gathered}
g(t)=\left(\frac{(a b)^{\nu} 2^{2 \mu} t^{2 \nu}}{\left(\lambda c^{a t}+1\right)\left(\lambda c^{b t}+1\right)}\right)^{\alpha} \frac{1+\lambda(-1)^{a+1} c^{a b t}}{\left(\lambda c^{a t}+1\right)\left(\lambda c^{b t}+1\right)} c^{a b(x+y) t+a^{2} b^{2} z t^{2}} \\
g(t)=\left(\frac{2^{\mu}(a t)^{\nu}}{\left(\lambda c^{a t}+1\right.}\right)^{\alpha} c^{a b x t+a^{2} b^{2} z t^{2}}\left(\frac{1-\lambda\left(c^{-b t}\right)^{a}}{\lambda c^{b t}+1}\right)\left(\frac{2^{\mu}(b t)^{\nu}}{\lambda c^{b t}+1}\right)^{\alpha} c^{a b y t}\left(\frac{1-\lambda\left(c^{-a t}\right)^{b}}{\lambda c^{a t}+1}\right) \\
(4.7)=\left(\frac{2^{\mu}(a t)^{\nu}}{\left(\lambda c^{a t}+1\right.}\right)^{\alpha} c^{a b x t+a^{2} b^{2} z t^{2}} \sum_{i=0}^{a-1}(-\lambda)^{i} c^{b t i}\left(\frac{2^{\mu}(b t)^{\nu}}{\lambda c^{b t}+1}\right)^{\alpha} c^{a b y t} \sum_{j=0}^{b-1}(-\lambda)^{j} c^{a t j}
\end{gathered}
$$




$$
\begin{gathered}
=\left(\frac{2^{\mu}(a t)^{\nu}}{\left(\lambda c^{a t}+1\right.}\right)^{\alpha} c^{a^{2} b^{2} z t^{2}} \sum_{i=0}^{a-1} \sum_{j=0}^{b-1}(-\lambda)^{i+j} c^{\left(b x+\frac{b}{a} i+j\right) a t} \sum_{k=0}^{\infty} F_{k}^{(\alpha)}(a y ; \lambda ; \mu, \nu, c) \frac{(b t)^{k}}{k !} \\
=\sum_{n=0}^{\infty} \sum_{i=0}^{a-1} \sum_{j=0}^{b-1}(-\lambda)^{i+j} F_{n}^{(\alpha)}\left(b x+\frac{b}{a} i+j, b^{2} z ; \lambda ; \mu, \nu, c\right) \frac{(a t)^{n}}{n !} \\
\sum_{k=0}^{\infty} F_{k}^{(\alpha)}(a y ; \lambda ; \mu, \nu, c) \frac{(b t)^{k}}{(k) !} \\
\left(b x+\frac{b}{a} i+j, b^{2} z ; \lambda ; \mu, \nu, c\right) F_{k}^{(\alpha)}(a y ; \lambda ; \mu, \nu, c)
\end{gathered}
$$

Since $(-1)^{a+1}=(-1)^{b+1}$, the expression for

$$
g(t)=\left(\frac{(a b)^{\nu} 2^{2 \mu} t^{2 \nu}}{\left(\lambda c^{a t}+1\right)\left(\lambda c^{b t}+1\right)}\right)^{\alpha} \frac{1+\lambda(-1)^{a+1} c^{a b t}}{\left(\lambda c^{a t}+1\right)\left(\lambda c^{b t}+1\right)} c^{a b(x+y) t+a^{2} b^{2} z t^{2}}
$$

is symmetric in a and b. Therefore, by symmetry we obtain the following power series expansion for $\mathrm{g}(\mathrm{t})$

$$
\begin{gathered}
g(t)=\sum_{n=0}^{\infty} \sum_{k=0}^{n}\left(\begin{array}{c}
n \\
k
\end{array}\right) \sum_{i=0}^{b-1} \sum_{j=0}^{a-1}(-\lambda)^{i+j} b^{n-k} a_{H}^{k} F_{n-k}^{(\alpha)} \\
\left(a x+\frac{a}{b} i+j, a^{2} z ; \lambda ; \mu, \nu, c\right) F_{k}^{(\alpha)}(b y ; \lambda ; \mu, \nu, c)
\end{gathered}
$$

By comparing the coefficients of $t^{n}$ on the right hand sides of the last two equations, we arrive at the desired result.

By setting $\lambda=-\lambda, \mu=0$ and $\nu=1$ in Theorem (4.2) and then multiplying $(-1)^{\alpha}$ on both sides of the result, we have the following corollary

Corollary 1. For any integral $n \geq 1, x, y \in R$ and $\alpha \in N$. Then

$$
\sum_{k=0}^{n}\left(\begin{array}{c}
n \\
k
\end{array}\right) \sum_{i=0}^{a-1} \sum_{j=0}^{b-1}(-\lambda)^{i+j} a^{n-k} b_{H}^{k} B_{n-k}^{(\alpha)}\left(b x+\frac{b}{a} i+j, b^{2} z ; \lambda ; c\right) B_{k}^{(\alpha)}(a y ; \lambda ; c)
$$

$$
=\sum_{k=0}^{n}\left(\begin{array}{c}
n \\
k
\end{array}\right) \sum_{i=0}^{b-1} \sum_{j=0}^{a-1}(-\lambda)^{i+j} a^{k} b^{n-k}{ }_{H} B_{n-k}^{(\alpha)}\left(a x+\frac{a}{b} i+j, a^{2} z ; \lambda ; c\right) B_{k}^{(\alpha)}(b y ; \lambda ; c)
$$


Remark. For $\lambda=1, c=e$ in equation (4.10), the result reduces to known result of Pathan and Khan [14] and further by taking $c=e, \lambda=1, \alpha=1$ in equation (4.10), the result reduces to another known result of Pathan [13].

By setting $\mu=1$ and $\nu=0$ in Theorem (4.2), we have the following corollary

Corollary 2. For any integral $n \geq 1, x, y \in R$ and $\alpha \in N$. Then

$$
\sum_{k=0}^{n}\left(\begin{array}{c}
n \\
k
\end{array}\right) \sum_{i=0}^{a-1} \sum_{j=0}^{b-1}(-\lambda)^{i+j} a^{n-k} b_{H}^{k} E_{n-k}^{(\alpha)}\left(b x+\frac{b}{a} i+j, b^{2} z ; \lambda ; c\right) E_{k}^{(\alpha)}(a y ; \lambda ; c)
$$

$$
=\sum_{k=0}^{n}\left(\begin{array}{c}
n \\
k
\end{array}\right) \sum_{i=0}^{b-1} \sum_{j=0}^{a-1}(-\lambda)^{i+j} a^{k} b^{n-k}{ }_{H} E_{n-k}^{(\alpha)}\left(a x+\frac{a}{b} i+j, a^{2} z ; \lambda ; c\right) E_{k}^{(\alpha)}(b y ; \lambda ; c)
$$

By setting $\mu=1$ and $\nu=1$ in Theorem (4.2), we have the following corollary

Corollary 3.For any integral $n \geq 1, x, y \in R$ and $\alpha \in N$. Then

$$
\sum_{k=0}^{n}\left(\begin{array}{c}
n \\
k
\end{array}\right) \sum_{i=0}^{a-1} \sum_{j=0}^{b-1}(-\lambda)^{i+j} a^{n-k} b_{H}^{k} G_{n-k}^{(\alpha)}\left(b x+\frac{b}{a} i+j, b^{2} z ; \lambda ; c\right) G_{k}^{(\alpha)}(a y ; \lambda ; c)
$$

$$
=\sum_{k=0}^{n}\left(\begin{array}{c}
n \\
k
\end{array}\right) \sum_{i=0}^{b-1} \sum_{j=0}^{a-1}(-\lambda)^{i+j} a^{k} b^{n-k}{ }_{H} G_{n-k}^{(\alpha)}\left(a x+\frac{a}{b} i+j, a^{2} z ; \lambda ; c\right) G_{k}^{(\alpha)}(b y ; \lambda ; c)
$$

\section{References}

[1] A. Erdelyi, W. Magnus, F. Oberhettinger and F. Tricomi, Higher transcendental functions, vols. 1-3, (1953).

[2] D. Q. Lu and Q. M. Luo, Some properties of the generalized Apostol tpe polynomials, Boundary Value Problems., (2013),2013:64.

[3] E. T. Bell, Exponential polynomials, Ann. of Math., 35(1943), 258-277.

[4] E. R. Hansen, A table of series and products, Printice Hall, Englewood Cliffs, NJ, (1975).

[5] F. Magnus, W. Oberhettinger and R. P. Soni, Some formulas and theorem for the special functions of mathematical physics, Third enlarged edition, Springer-Verlag, New York, (1966). 
[6] G. Dattoli, S. Lorenzutt and C. Cesarano, Finite sums and generalized forms of Bernoulli polynomials,Rendiconti di Mathematica, 19(1999), 385-391.

[7] H. M. Srivastava and H. L .Manocha, A treatise on generating functions, Ellis Horwood Limited. Co. New York, (1984).

[8] H. M. Srivastava, M. Garg and S. Choudhary, A new generalization of the Bernoulli and related polynomials, Russian J. Math. Phys, 17(2010), 251-261.

[9] H. M. Srivastava, M. Garg and S. Choudhary, Some new families of generalized Euler and genochhi polynomials, Taiwanese J. Math, 15(1)(2011), 283-305.

[10] H. Yang, An identity of symmetry for the Bernoulli polynomials, Discrete Math. (2007)dol:10:10,16/j.disc 2007.03.030.

[11] L. Comlet, The art of finite and infinite expansions, (Translated from french by J. W. Nilenhuys), Reidel, Dordrecht, 1974.

[12] M. Abramowitz and I. A. Stegun, Handbook of mathematical functions with formulas graphs and mathematical tables, National Bureau of Standards, Washington, DC, 1964.

[13] M. A. Pathan, A new class of generalized Hermite-Bernoulli polynomials, Georgian Mathematical Journal, 19(2012), 559-573.

[14] M. A. Pathan and W. A. Khan, Some implicit summation formulas and symmetric identities for the generalized Hermite-Bernoulli polynomials, Mediterr.J.Math.(2014),DOI 10.1007/s00009-014-0423-0, Springer Basel 2014.

[15] Q. M. Luo, Apostol Euler polynomials of higher order and gaussian hypergeometric functions, Taiwanese J. Math., 10(4)(2006), 917-925.

[16] Q. M. Luo, q-extensions for the Apostol-Genocchi polynomials, Gen. Math., 17(2)(2009), 113-125.

[17] Q. M. Luo, Extensions for the Genocchi polynomials and its fourier expansions and integral representations, Osaka j. Math., 48(2011), 291-310.

[18] Q. M. Luo and H. M. Srivastava Some generalizations of the Apostol-Bernoulli and Apostol-Euler polynomials, J. Math. Anal. Appl, 308(1)(2005), 290-302.

[19] Q. M. Luo and H. M. Srivastava, Some generalizations of the Apostol Genocchi polynomials and the stirling number of the second kind, Appl. Math. Comput, 217(2011), 5702-5728.

[20] Q. M. Luo and H. M. Srivastava, Some relationships between the Apostol-Bernoulli and Apostol-Euler polynomials, Comput. Math. Appl, 51(2006), 631-642.

[21] S. Khan, M. A. Pathan, N. A. M. Hassan, G. Yasmin , Implicit summation formula for Hermite and related polynomials, J. Math. Anal. Appl, 344(2008), 408-416.

[22] S. L. Yang and Z. K. Qiao, Some symmetry identities for the Euler polynomials, J. Math. Resrch. Exposition, 30(3)(2010), 457-464.

[23] Y. Luke, The special functions and their approximations, vols, 1-2, 1969.

[24] Yu. A. Brychkov, On multiple sums of special functions, Integral Trans. Spec. Func., 21(12)(2010), 877-884.

[25] Z. Zhang and H. Yang, Several identities for the generalized Apostol Bernoulli polynomials, Computers and Mathematics with Applications, 56(2008), 2993-2999. 